\title{
Reoperation Rates after Laminoplasty for Cervical Disorders: A 26-Year Period Survival Function Method Analysis
}

\author{
Ko Hashimoto ${ }^{12)}$, Toshimi Aizawa ${ }^{1)}$, Hiroshi Ozawa ${ }^{3)}$, Yasuhisa Tanaka ${ }^{4)}$, Takashi Kusakabe ${ }^{5}$, Naoki Morozumi ${ }^{6}$, \\ Yutaka Koizumi ${ }^{6}$, Tetsuro Sato ${ }^{7}$, Hironori Hyodo ${ }^{7)}$, Tomowaki Nakagawa ${ }^{7}$, Eiji Takahashi ${ }^{7}$, Takeshi Hoshikawa ${ }^{4)}$, \\ Hideki Imaizumi ${ }^{8)}$, Shinji Ogawa ${ }^{9)}$, Fumio Kasama ${ }^{10)}$, Haruo Kanno ${ }^{1)}$, Eiji Itoi ${ }^{1)}$ and Shoichi Kokubun ${ }^{11}$
}

1) Department of Orthopaedic Surgery, Tohoku University Graduate School of Medicine, Sendai, Japan

2) Department of Orthopaedic Surgery, Takeda General Hospital, Fukushima, Japan

3) Department of Orthopaedic Surgery, Tohoku Medical and Pharmaceutical University, Sendai, Japan

4) Department of Orthopaedic Surgery, Tohoku Central Hospital, Yamagata, Japan

5) Department of Orthopaedic Surgery, Tohoku Rosai Hospital, Sendai, Japan

6) Department of Orthopaedic Surgery, Sendai Nishitaga Hospital, Sendai, Japan

7) Department of Orthopaedic Surgery, Sendai Orthopaedic Hospital, Sendai, Japan

8) Department of Orthopaedic Surgery, Osaki Citizen Hospital, Miyagi, Japan

9) Department of Orthopaedic Surgery, Sendai Medical Center, Sendai, Japan

10) Department of Orthopaedic Surgery, Matsuda Hospital, Sendai, Japan

11) Research Center for Spine and Spinal Cord Disorders, Sendai Nishitaga Hospital, Sendai, Japan

\begin{abstract}
:
Introduction: Laminoplasty is a common surgery for cervical myelopathy. Previous studies have analyzed the reoperation rates in posterior decompression surgeries of the cervical spine. However, few studies have solely focused on midlinesplitting laminoplasty (MSL) using a large number of patients. This aims to analyze the reoperation rates after MSL using the survival function method.

Methods: Between 1988 and 2013, 4,208 MSLs were performed as a primary operation for cervical myelopathy and enrolled in our spinal surgery registration system. The Kaplan-Meier survival function method was used to analyze the rates of reoperation.

Results: Of 4,208 patients with primary MSL, 40 underwent reoperation for neurological complications. The overall reoperation rate was $0.26 \%, 0.64 \%, 0.83 \%, 0.93 \%$, and $0.95 \%$ at $1,5,10,20$, and $>20$ years, respectively. The causes of reoperation were postoperative cervical radiculopathy in 10 patients, stenosis at an adjacent level in 8 , stenosis due to failed "open-door" lamina in 6 , instability of the cervical spine in 4 , cervical disc herniation in 3 , elongation of ossification of the posterior longitudinal ligament in 3, spinal cord injury in 1 , fracture of the cervical spine in 1 , postoperative scar formation in 1, ossification of anterior longitudinal ligament in 1, and unknown in 2. The number of patients with surgical site infection (SSI) who needed surgical debridement was $34(0.81 \%)$.

Conclusions: Excluding reoperations for SSI, the reoperation rate of MSL was approximately $1.0 \%$ at the maximum of 26 years after surgery. MSL was determined to be a reliable surgical procedure regarding postoperative complications requiring additional surgeries.
\end{abstract}

Keywords:

Cervical myelopathy, midline-splitting laminoplasty, reoperation rate, survival function method analysis 


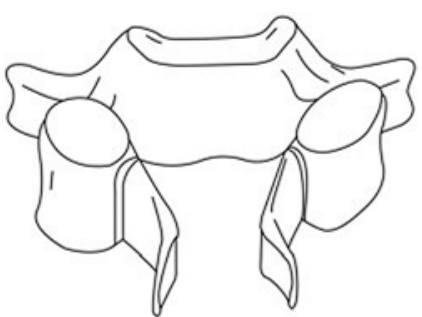

a

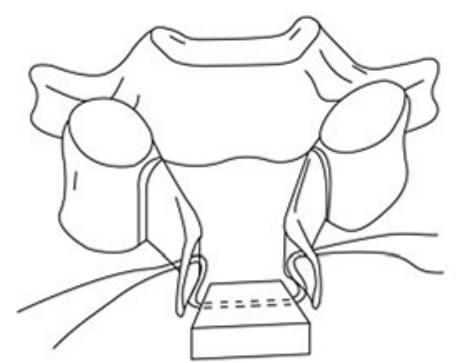

b

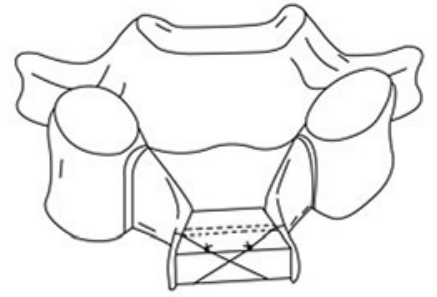

C

Figure 1. Operative method of the midline-splitting laminoplasty. (a) Midline-splitting and opening of the lamina. (b) Installing a rhomboid-shaped hydroxyapatite spacer to the opened lamina with double threads. (c) Cross-multiplied ties fixing the hydroxyapatite spacer to the opened lamina.

\section{Introduction}

Cervical posterior decompression has been utilized worldwide to relieve spinal cord compression mainly due to spondylotic changes and the ossification of the posterior longitudinal ligament (OPLL). The ideal operative method is one that achieves complete decompression of the spinal cord while preserving as much spinal stability as possible with fewer surgery-related complications. The commonly used techniques are laminectomy, laminoplasty, and laminectomy with instrumented fusion. Laminectomy causes mechanical vulnerability of the cervical spine compared with other techniques ${ }^{1,2}$. To preserve the stability of the cervical spine, various methods of laminoplasty have been developed over the last several decades, mainly in $\mathrm{Japan}^{3}$ because this population has higher incidences of cervical myelopathy due to an innately narrower spinal canal ${ }^{4)}$ and a high prevalence of OPLL $^{5,6)}$. Among these, two major techniques have been widely employed: the midline-splitting and open-door meth$\mathrm{ods}^{7}$. Another option producing excellent results is laminectomy with instrumented fusion ${ }^{8}$. However, our group has a policy to use less instruments during spinal surgery, unless a superior outcome is expected with instrumented surgery". Therefore, since 1988, our group has been primarily using the midline-splitting laminoplasty (MSL) technique for cervical myelopathy with multilevel stenosis.

Reoperations are usually performed when patients experience unfavorable postoperative events, such as recurrent symptoms and surgical site infection (SSI) after the primary surgery. Several reports have characterized the reoperation rates after primary posterior decompression surgeries for cervical spine disorders. However, in most of these reports, size of the data set or the length of follow-up was limited $^{10-12)}$. Additionally, the reoperation rates were calculated by dividing the number of reoperations by the total number of primary surgeries during the study period. Survival function methods, such as the Kaplan-Meier method and Cox proportional hazards model, should be employed to determine the actual reoperation rates. Our group has performed more than 60,000 spinal surgeries between 1988 and 2013 .
Using this database, our group published the reoperation rates of the lumbar spine disorders by Kaplan-Meier methods $^{9,13}$. We aimed to elucidate the utility of MSL by calculating the actual reoperation rates using survival function method analysis in this study. In addition, the pathologies leading to reoperation were evaluated.

\section{Surgical procedure of our midline-splitting laminoplasty $(M S L)^{(4)}$}

MSL was originally developed by Kurokawa ${ }^{15}$. Our group has been using the modified method using hydroxyapatite spacers instead of autologous iliac bone grafts as in the original method. Our surgical procedure of MSL is performed as follows (Fig. 1). In a prone position, the cervical laminae are exposed with a mid-line skin incision, and the spinous processes are trimmed at a height of approximately $10 \mathrm{~mm}$. Longitudinal gutters are created at the border of laminae and lateral masses using a 4-mm high-speed burr on one side. The mid-line of the spinous processes is split with a 2-mm high-speed burr or a thread-wire saw ${ }^{16}$. While checking the elasticity of the hinges, gutters on the other side are created. The split laminae are then opened like French doors (Fig. 1a). A hole is generated in each laminar flap with a 2-mm high-speed burr to pass threads through for spacer fixation (Fig. 1b). A trapezoidal hydroxyapatite spacer with a porous structure is installed between each split lamina with cross-multiplied ties using non-absorbable threads to stabilize the opened laminae (Fig. 1c). This procedure is applied from $\mathrm{C} 3$ to $\mathrm{C} 6$, basically, unless the decompression of $\mathrm{C} 2$ or $\mathrm{C} 7$ level is necessary to guarantee a thorough decompression of the spinal cord. The C3 to C6 laminoplasty can achieve a thorough decompression of the spinal cord in most myelopathy cases because the frequency of spinal cord compression at the C6-7 disc level is only $6 \%{ }^{177}$.

\section{Materials and Methods}

Spine surgeries performed in the orthopedic departments in Miyagi Prefecture, located in northeastern Japan with a 
Table 1. Demographic Data of Patients Who Had Undergone Midline Splitting Laminoplasty (Msl) as a Primary Surgery for Cervical Myelopathy in Miyagi Prefecture between 1988 and 2013.

\begin{tabular}{lcc}
\multicolumn{4}{c}{ A. Number and average age of patients } \\
\hline Gender & Number of patients & Average age at surgery (years) \\
\hline Male & 2,868 & 61 (range: $17-94)$ \\
Female & 1,340 & 65 (range: $21-92)$ \\
Total & 4,208 & 62 (range: $17-94)$ \\
\hline
\end{tabular}

\begin{tabular}{lc}
\hline \multicolumn{2}{l}{ B. Age distribution } \\
\hline \multicolumn{1}{c}{ Age } & Number of patients \\
\hline$\leq 19$ & 2 \\
$20-29$ & 11 \\
$30-39$ & 105 \\
$40-49$ & 456 \\
$50-59$ & 974 \\
$60-69$ & 1,390 \\
$70-79$ & 1,052 \\
$\geq 80$ & 214 \\
Unknown & 4 \\
\hline
\end{tabular}

population of approximately 2.33 million in 2013 , have been recorded into our spine registry since $1988^{18,19}$. The registry contains surgical information, such as operated spinal level" (cervical, thoracic, lumbar, and/or sacral), main pathology, and operative methods in addition to patient-identifying information. Of the total registration of more than 60,000 spine surgeries, approximately 35,000 were solely performed in Miyagi Prefecture, in which 8,033 were for cervical spine disorders. Of these, 4,208 were MSL for cervical myelopathy as a primary surgery for cervical myelopathy. In our group, MSL has been performed for patients with cervical myelopathy whose neurological deficit is specified between $\mathrm{C} 2$ and $\mathrm{C} 7$, corresponding to the spinal factors found in the imaging studies, such as magnetic resonance imaging and myelography. The spinal factors include developmental spinal canal stenosis, osteophyte of the vertebral body, degenerative spondylolisthesis, OPLL, disc herniation, ossification of the ligamentum flavum, and so on. We first analyzed the following epidemiological characteristics of this procedure during the 26 years using the data from our spine registry: the number of surgeries and age distribution of the patients. This study was performed solely based on the database and operation records of the patients that did not include followup information of the patients. First, patients who underwent multiple cervical surgeries after primary MSL were selected from the database by collating the identity of the patients. Thereafter, their operation records were collected to analyze the surgical procedures, operated spinal levels, causes of reoperation, and other salient information. The reoperations for neurological complications and SSI were separately analyzed.

The 26-year overall reoperation rates of the primary MSLs were analyzed using the Kaplan-Meier survival func- tion method as previously described ${ }^{20)}$. The interval between the primary operation and reoperation was calculated by subtracting the date of the primary surgery from that of reoperation and indicated in years for reoperations for neurological complications and days for SSI. Statistical analyses were performed using Microsoft Excel software.

This research has been approved by the IRB of the authors' affiliated institutions.

\section{Results}

The demographic data of 4,208 patients who underwent MSL as a primary decompression surgery are shown in Table 1 . The male/female ratio was 2.2 , and the average age at the primary surgery was $62 \pm 11$ years. As for the age distribution, the mode was in the seventh decade. A total of $36 \%$ patients were $\geq 60$ years old. The annual changes in the number of MSLs as primary surgery are shown in Fig. 2a. The number of MSLs had been on an increasing trend from 1988 to 2013. The annual changes in the age distribution of patients who underwent MSL as a primary surgery are shown in Fig. $2 \mathrm{~b}$. The ratios of patients $\geq 70$ years old gradually increased throughout the 26-year study period. In 2013 , approximately one-third of the patients were $\geq 70$ years old and $8 \%$ were $\geq 80$ years old.

Of 4,208 patients with primary MSL, 40 underwent reoperation for neurological complications. The details of the patients with reoperation are summarized in Table 2. The spinal levels of the primary surgeries were $\mathrm{C} 3-6$ in 25 patients, C4-6 in 5, C3-7 in 3, C2-6 in 1, C3-4 in 1, C5-6 in 1, and unknown in 4 . The causes of reoperation were postoperative cervical radiculopathy in 10 patients, stenosis at an adjacent level in 8, stenosis due to failed "open-door" lamina in 6, instability of the cervical spine in 4 , cervical disc herniation in 3 , elongation of OPLL in 3, spinal cord injury in 1, fracture of the cervical spine in 1, postoperative scar formation in 1, ossification of anterior longitudinal ligament in 1, and unknown in 2. Stenosis due to failed "open-door" lamina included the spinal cord compression due to fractured "opendoor" lamina sinking into the spinal canal and displaced spacers causing re-closure of the lamina (Fig. 3). Among the 10 patients with postoperative cervical radiculopathy, two had C5 palsy and were reoperated at 21 and 52 days after the primary operations. The remaining patients who were reoperated for radiculopathy arose at $\mathrm{C} 6 / 7$ or $\mathrm{C} 7 / \mathrm{T} 1$, which is out of the range of laminoplasty. Except those for C5 palsy, the reoperations for postoperative cervical radiculopathy were performed at a range of 1.3-12.0 years after the primary surgeries. The reoperation procedures were laminectomy in 13 patients, foraminotomy in 9, anterior spinal fusion (ASF) in 8, MSL in 5, laminectomy with posterior fusion in 3, and other operations in 2 . The average interval between primary MSL and reoperation was 4.6 (range, 0-23) years.

The Kaplan-Meier analysis data of the overall reoperation rate during the 26-year period is shown in Fig. 4 and is as 
a
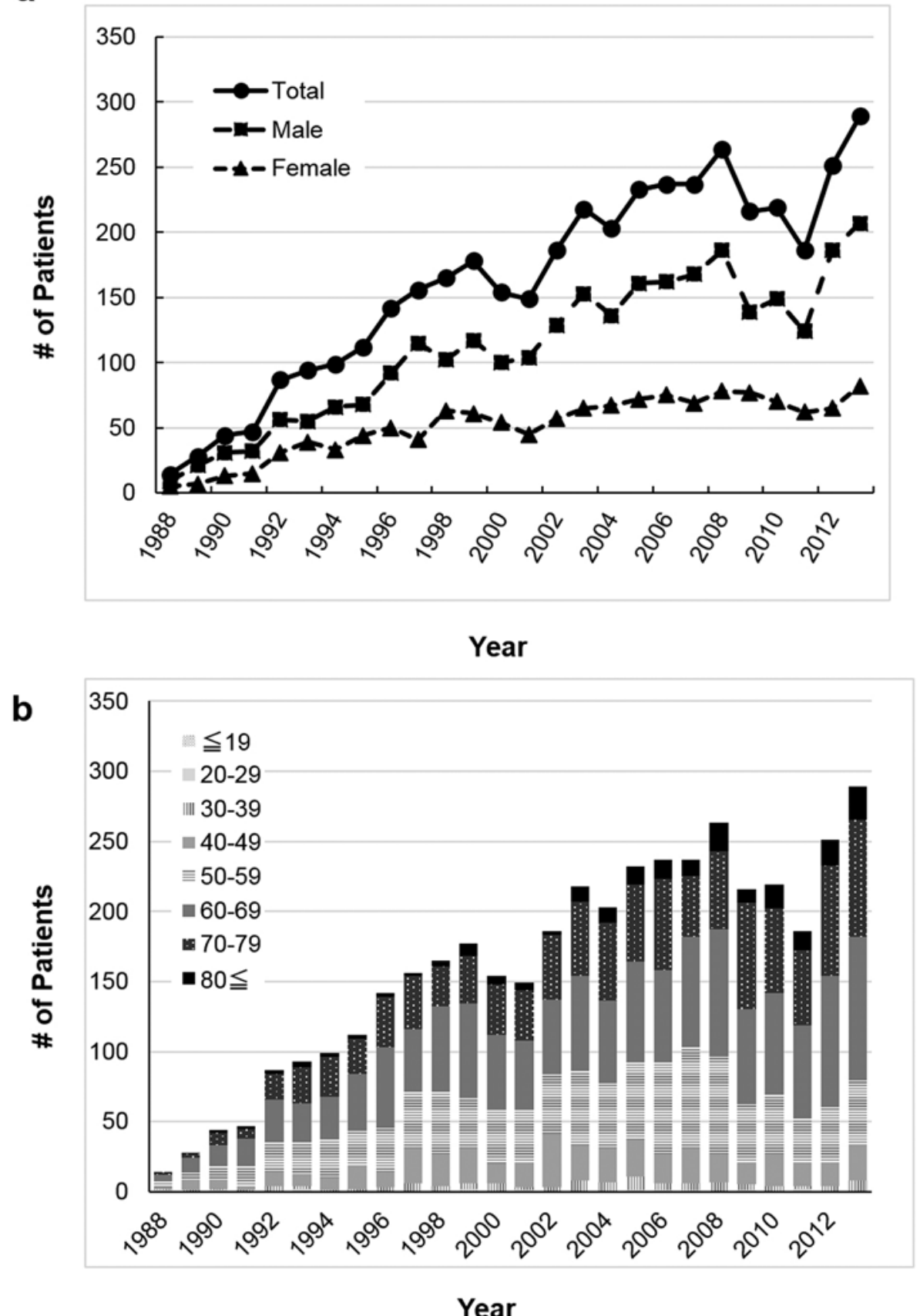

Figure 2. Annual changes in the number (a) and the age distribution (b) of patients who underwent the midline-splitting laminoplasty as the primary surgery for cervical myelopathy in Miyagi Prefecture, Japan.

follows: $0.26 \%, 0.50 \%, 0.64 \%, 0.83 \%, 0.88 \%, 0.93 \%$, and $0.95 \%$ at $1,3,5,10,15,20$, and $>20$ years, respectively.

The number of patients with SSI after primary MSL who underwent surgical debridement was $34(0.81 \%)$. The average duration between primary MSL and surgical debridement was 42 (range, 0-504) days. Within 30 days after primary surgeries, 27 patients had surgical debridement.

\section{Discussion}

The number of MSLs has been dramatically increasing in our group. From the start of our spine registry in 1988, the number of MSLs has increased from 15 in 1988 to more than 300 in 2013. Japan has one of the most aged populations in the world. The Japanese Ministry of Internal Affairs and Communications has reported that approximately $25 \%$ of the Japanese population was older than 65 years in $2013^{20}$. For other degenerative spinal disorders, the number of operations for cervical myelopathy has been increasing in recent years ${ }^{9}$. With similar clinical results as ASF, a recent study of our group indicated that cervical laminoplasty can be employed even for myelopathy caused by single-level disc herniation ${ }^{21}$. Spine surgeons have recently tended to prefer posterior surgeries because these surgeries have fewer 
Table 2. Characteristics of the Patients with Reoperation.

A. Number and average age of patients at surgery

\begin{tabular}{lcc}
\hline Gender & Number of patients & $\begin{array}{c}\text { Average age at primary } \\
\text { laminoplasty (years) }\end{array}$ \\
\hline Male & 31 & 56 (range: $34-71)$ \\
Female & 9 & 64 (range: $43-76)$ \\
Total & 40 & 58 (range: $34-76)$ \\
\hline
\end{tabular}

B. Number of revision surgeries by durations after primary surgery

Duration between primary and revision surgery Number of surgeries

\begin{tabular}{lc}
$\leq 1$ year & 10 \\
$1-3$ years & 11 \\
$3-5$ years & 6 \\
$5-10$ years & 8 \\
$10-15$ years & 2 \\
$15-20$ years & 2 \\
$\geq 20$ years & 1 \\
\hline
\end{tabular}

Average 4.6 years (range: 22 days-23.3 years)

risks of critical complications than those expected in cervical anterior surgeries, such as retropharyngeal edema and esophageal damage ${ }^{22}$.

The reoperation rates of cervical posterior decompression have been reported to be $0.4 \%-3.8 \%^{11,12,23)}$. For the reoperation rates of laminoplasty, Ha et al. ${ }^{23)}$ analyzed 339 cases for cervical OPLL and reported a reoperation rate of $3.8 \%$ with a mean follow-up period of 26 months. Lee et al. ${ }^{24)}$ analyzed 145 cases with a Cox proportional-hazards regression survivorship curve and reported the overall reoperation rates for adjacent segment disorder as approximately $4 \%$ at 10 years. In addition, meta-analyses have been performed by several groups $^{11,12)}$. With follow-up periods between 0.25 and 12 years, Jiang et al. ${ }^{11)}$ performed a meta-analysis of 383 cases and reported a reoperation rate of $1.6 \%$. Liu et al. ${ }^{12)}$ reported a reoperation rate of $0.9 \%$. The number of patients was less and the follow-up period was relatively short in these studies. To clarify the actual reoperation rates of a particular surgical method, a long-term follow-up with larger number of patients with the same surgical procedure is necessary. Furthermore, survival function methods, such as the KaplanMeier method or Cox proportional hazards model, should be utilized.

Our group has published the reoperation rates of lumbar spinal disorders with survival function methods using our spine registry ${ }^{13,20)}$. In the present study, we analyzed the reoperation rates of MSL in the largest number of patients and the longest follow-up period to date, to the best of our knowledge. From the analysis of 4,208 MSLs with a maximum follow-up period of 26 years, the reoperation rates of MSL were demonstrated to reach a plateau of approximately $1.0 \%$ at 15 years after the primary surgery. The overall reoperation rates were close to the $0.9 \%-1.6 \%$ rates previously reported $^{11,12)}$. Low reoperation rates of MSL were confirmed with the largest and the longest survival function study.

The low reoperation rates could ascribe advantages of a

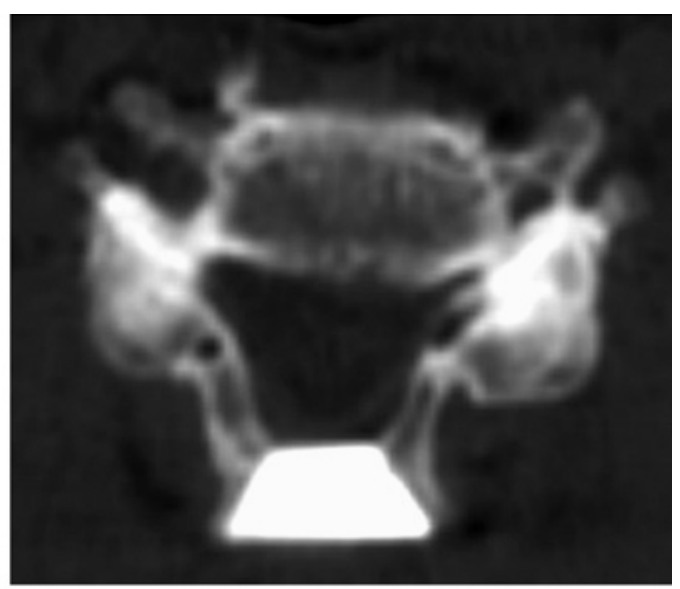

b

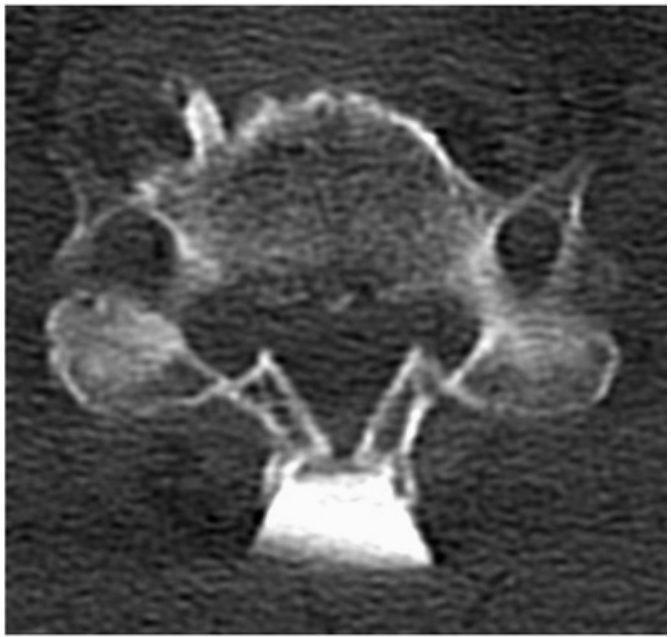

C

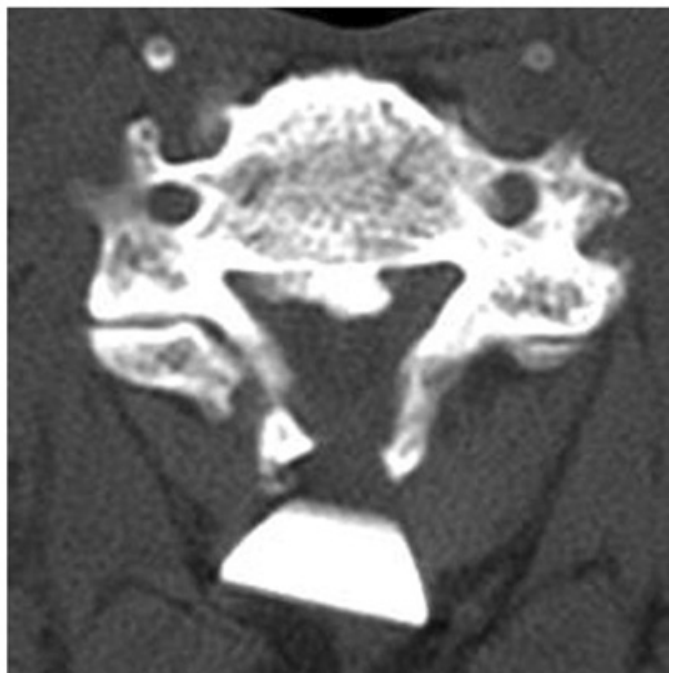

Figure 3. Computed tomograms of the "open-door" lamina after midline-splitting laminoplasty. (a) Ideally opened lamina with a rhomboid-shaped hydroxyapatite spacer. (b) Fractured "open-door" lamina sinking toward spinal canal. (c) Displaced hydroxyapatite spacer causing closure of the opened lamina.

MSL in achieving multilevel decompression, including potential stenotic segments. However, the low reoperation rate indicated fewer patients after MSL had secondary neurological deterioration requiring another surgical intervention. It does not demonstrate that MSL can provide better clinical results than other procedures in terms of postoperative 


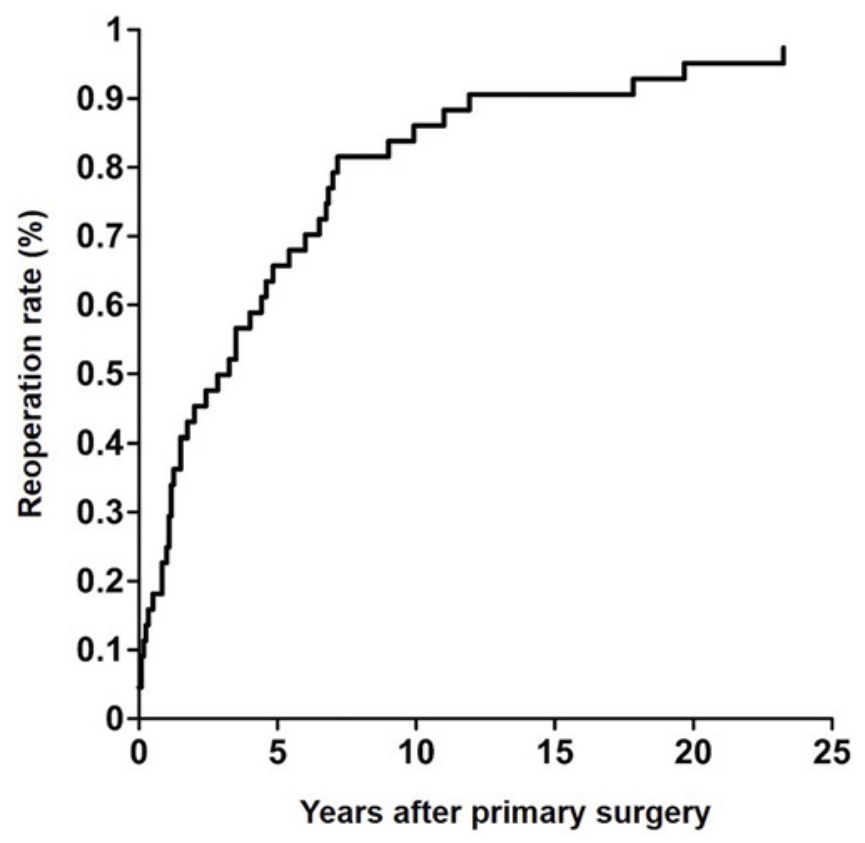

Figure 4. Overall reoperation rate of the midline-splitting laminoplasty calculated by the Kaplan-Meier method. The cumulative reoperation rate steadily increased up to $0.9 \%$ at 10 years after primary surgery before reaching a sustained plateau afterward.

symptoms, such as neck pain, which do not result in reoperation.

Cervical laminectomy, laminoplasty, posterior instrumentation, and their combination are widely accepted methods to achieve multilevel decompression of the spinal cord. For laminectomy, the postoperative hypermobility of the cervical spine is expected due to the removal of the posterior segments, which differs from laminoplasty or posterior decompression with instrumented fusion ${ }^{1,7)}$. Various methods of laminoplasty have been developed and achieved favorable postoperative results in $\mathrm{Japan}^{7)}$. Postoperative cervical kyphosis can be best avoided with posterior instrumentation $^{25}$. However, instrumented fusion has risks of instrumentation failure or persistent discomfort of the autograft harvest site ${ }^{26}$. The basic policy of our group for spine surgeries has been "less invasive, less complicated, less fusion, less metal work, and less expensive."9) Thus, our group has been utilizing laminoplasty as a first-choice surgery for cervical myelopathy with multilevel stenosis in recent years. In the most recent 5 years (2010-2014), 67\% patients with a surgery for cervical myelopathy were treated with MSL in our affiliated hospitals (data not shown).

Postoperative complications of laminoplasty, including those without reoperation, have been reported in several studies. Liu et al. ${ }^{12)}$ performed a meta-analysis of 321 cases and reported that postoperative kyphosis occurred in $11.8 \%$, C5 palsy in $9.6 \%$, axial neck pain in $9.0 \%$, and neurological deficits in $2.2 \%$ patients. Only 3 patients $(0.9 \%)$ underwent reoperation because of neurological deterioration due to new disc herniation, restenosis caused by collapsed laminoplasty, and postoperative hematoma. Zhu et al. ${ }^{27)}$ reported in their meta-analysis of 285 cervical posterior surgeries that only one patient underwent reoperation because of cervical radiculopathy due to postoperative disc herniation. In the present study, the major causes of reoperation were cervical radiculopathy and myelopathy caused by an adjacent segment disorder, failed "open-door" lamina, disc herniation, and OPLL. Remarkably, reoperation for C5 palsy was performed only in two patients. The actual prevalence of postlaminoplasty C5 palsy is assumed to be much higher than the reoperation rate because it can conservatively recover in most cases $^{28)}$.

For SSI after cervical posterior surgeries, higher SSI rates (1\%-15\%) have been reported with instruments compared to laminectomy or laminoplasty $(0 \%-7 \%)^{29)}$. Conversely, the actual reoperation rate in laminoplasty has not been elucidated so far, because of the low frequency of severe SSI requiring surgical treatment and the limited number of samples. Our study demonstrated an actual reoperation rate of $0.81 \%$ for SSI in cervical laminoplasty. This rate was higher than that of our lumbar posterior decompression surgeries without instrumentation $(0.28 \%)^{30)}$. The installation of hydroxyapatite spacers, i.e., foreign bodies, or dead space formation around the spacers, might be related to the higher infection rate.

The present study has major limitations as a retrospective study using a multicenter database. The quality and reliability of data are not always guaranteed because of inaccurate registration or incomplete or loss of data in a long-term, multicenter study. We experienced difficulties in data collection, particularly for old medical records that had been abandoned or lost. In fact, the causes for reoperation in two patients could not be evaluated. Moreover, the clinical data, such as Japanese Orthopedic Association score for cervical myelopathy, have not been collected in the series of this database. In addition, some patients might have had reoperations out of our affiliated hospitals who could not be properly followed up. Because the population of patients with cervical myelopathy is relatively old, it is assumed that some patients died or became too old or frail for reoperations even when they had secondary complications.

\section{Conclusions}

Laminoplasty is a widely accepted procedure for cervical myelopathy. As shown in the Kaplan-Meier analysis, the reoperation rate of our MSL, excluding those for SSI, is $1.0 \%$ at a maximum of 26 years after surgery. Thus, MSL is determined to be a reliable procedure in terms of secondary complications requiring reoperations from this large-sized, long-term study.

Conflicts of Interest: The authors declare that there are no relevant conflicts of interest.

\section{Author Contributions:}

Ko Hashimoto: Study designing, data collection, data 
analysis, drafting and revising manuscripts

Toshimi Aizawa: Study designing, data collection, proof reading

Hiroshi Ozawa: Study designing, data collection

Yasuhisa Tanaka: Data collection

Takashi Kusakabe: Data collection

Naoki Morozumi: Data collection

Yutaka Koizumi: Data collection

Tetsuro Sato: Data collection

Hironori Hyodo: Data collection

Tomowaki Nakagawa: Data collection

Eiji Takahashi: Data collection

Takeshi Hoshikawa: Data collection

Hideki Imaizumi: Data collection

Shinji Ogawa: Data collection

Fumio Kasama: Data collection

Haruo Kanno: Data collection, proof reading

Eiji Itoi: Data collection

Shoichi Kokubun: Study designing, data collection

\section{References}

1. Della Pepa GM, Roselli R, La Rocca G, et al. Laminoplasty is better of laminectomy in cervical stenotic myelopathy: myth or truth? Eur Rev Med Pharmacol Sci. 2014;18(1 Suppl):50-4.

2. Lao L, Zhong G, Li X, et al. Laminoplasty versus laminectomy for multi-level cervical spondylotic myelopathy: a systematic review of the literature. J Orthop Surg Res. 2013;8:45.

3. Yonenobu K, Oda T. Posterior approach to the degenerative cervical spine. Eur Spine J. 2003;12 (Suppl 2):S195-201.

4. Chazono M, Tanaka T, Kumagae Y, et al. Ethnic differences in pedicle and bony spinal canal dimensions calculated from computed tomography of the cervical spine: a review of the Englishlanguage literature. Eur Spine J. 2012;21(8):1451-8.

5. Matsunaga S, Sakou T. Ossification of the posterior longitudinal ligament of the cervical spine: etiology and natural history. Spine (Phila Pa 1976). 2012;37(5):E309-14.

6. Wu JC, Liu L, Chen YC, et al. Ossification of the posterior longitudinal ligament in the cervical spine: an 11-year comprehensive national epidemiology study. Neurosurg Focus. 2011;30(3):E5.

7. Kurokawa R, Kim P. Cervical laminoplasty: the history and the future. Neurol Med Chir (Tokyo). 2015;55(7):529-39.

8. Lee CH, Lee J, Kang JD, et al. Laminoplasty versus laminectomy and fusion for multilevel cervical myelopathy: a meta-analysis of clinical and radiological outcomes. J Neurosurg Spine. 2015;22(6): 589-95.

9. Aizawa $\mathrm{T}$, Kokubun $\mathrm{S}$, Ozawa $\mathrm{H}$, et al. Increasing incidence of degenerative spinal diseases in Japan during 25 years: The registration system of spinal surgery in Tohoku University Spine Society. Tohoku J Exp Med. 2016;238(2):153-63.

10. Highsmith JM, Dhall SS, Haid RW, Jr., et al. Treatment of cervical stenotic myelopathy: a cost and outcome comparison of laminoplasty versus laminectomy and lateral mass fusion. J Neurosurg Spine. 2011;14(5):619-25.

11. Jiang L, Tan M, Dong L, et al. Comparison of anterior decompression and fusion with posterior laminoplasty for multilevel cervical compressive myelopathy: a systematic review and meta-analysis. J Spinal Disord Tech. 2015;28(8):282-90.

12. Liu X, Min S, Zhang H, et al. Anterior corpectomy versus posterior laminoplasty for multilevel cervical myelopathy: a systematic review and meta-analysis. Eur Spine J. 2014;23(2):362-72.
13. Aizawa T, Ozawa H, Kusakabe T, et al. Reoperation for recurrent lumbar disc herniation: a study over a 20-year period in a Japanese population. J Orthop Sci. 2012;17(2):107-13.

14. Kokubun S, Sato T. Cervical myelopathy and its management. Curr Orthop. 1998;12:7-12.

15. Kurokawa T. Midline-splitting cervical laminoplasty. Bessatsu Seikeigeka. 1982;2:234-40. Japanese.

16. Tomita K, Kawahara N, Toribatake Y, et al. Expansive midline Tsaw laminoplasty (modified spinous process-splitting) for the management of cervical myelopathy. Spine (Phila Pa 1976). 1998;23 (1):32-7.

17. Kokubun S. Neurological localization of the symptomatic level of lesion in cervical spondylotic myelopathy. Rinsho Seikeigeka. 1984;19:417-24. Japanese.

18. Kokubun S, Sato T, Ishii Y, et al. Cervical myelopathy in the Japanese. Clin Orthop Relat Res. 1996(323):129-38.

19. Sato T, Kokubun S, Tanaka Y, et al. Thoracic myelopathy in the Japanese: epidemiological and clinical observations on the cases in Miyagi Prefecture. Tohoku J Exp Med. 1998;184(1):1-11.

20. Aizawa $T$, Ozawa $H$, Kusakabe $T$, et al. Reoperation rates after fenestration for lumbar spinal canal stenosis: a 20 -year period survival function method analysis. Eur Spine J. 2015;24(2):381-7.

21. Koakutsu T, Morozumi N, Ishii Y, et al. Anterior decompression and fusion versus laminoplasty for cervical myelopathy caused by soft disc herniation: a prospective multicenter study. J Orthop Sci. 2010;15(1):71-8.

22. Rhee JM, Basra S. Posterior surgery for cervical myelopathy: laminectomy, laminectomy with fusion, and laminoplasty. Asian Spine J. 2008;2(2):114-26.

23. Ha Y, Moon BJ, You NK, et al. Clinical characteristics and surgical outcome of revision surgery in patients with cervical ossification of the posterior longitudinal ligament. World Neurosurg. 2016;90:164-71.

24. Lee JC, Lee SH, Peters C, et al. Risk-factor analysis of adjacentsegment pathology requiring surgery following anterior, posterior, fusion, and nonfusion cervical spine operations: survivorship analysis of 1358 patients. J Bone Joint Surg Am. 2014;96(21): 1761-7.

25. Woods BI, Hohl J, Lee J, et al. Laminoplasty versus laminectomy and fusion for multilevel cervical spondylotic myelopathy. Clin Orthop Relat Res. 2011;469(3):688-95.

26. Heller JG, Edwards CC, 2nd, Murakami H, et al. Laminoplasty versus laminectomy and fusion for multilevel cervical myelopathy: an independent matched cohort analysis. Spine (Phila Pa 1976). 2001;26(12):1330-6.

27. Zhu B, Xu Y, Liu X, et al. Anterior approach versus posterior approach for the treatment of multilevel cervical spondylotic myelopathy: a systemic review and meta-analysis. Eur Spine J. 2013;22 (7):1583-93.

28. Hirai T, Okawa A, Arai Y, et al. Middle-term results of a prospective comparative study of anterior decompression with fusion and posterior decompression with laminoplasty for the treatment of cervical spondylotic myelopathy. Spine (Phila Pa 1976). 2011;36 (23):1940-7.

29. Yoon ST, Hashimoto RE, Raich A, et al. Outcomes after laminoplasty compared with laminectomy and fusion in patients with cervical myelopathy: a systematic review. Spine (Phila Pa 1976). 2013;38(22 Suppl 1):S183-94.

30. Aizawa T, Ozawa H, Kusakabe T, et al. Prevalence of reoperations for perioperative complications in lumbar sipnal surgery. J Spine Res. 2012;3(2):149-53. 
Spine Surgery and Related Research is an Open Access journal distributed under the Creative Commons Attribution-NonCommercial-NoDerivatives 4.0 International License. To view the details of this license, please visit (https://creativeco mmons.org/licenses/by-nc-nd/4.0/) 